\title{
LAS FUNDACIONES EMPRESARIALES EN EL CUMPLIMIENTO DE LOS ODS: UNA MIRADA DESDE LOS SECTORES DE MINERÍA E HIDROCARBUROS
}

\author{
Ángela Sofía MunÉvar*
}

\section{Resumen}

La implementación de los objetivos de desarrollo sostenible (oDs) requiere de la participación de actores externos al gobierno, como los sectores privado y social. A partir de ello, se realiza un análisis sobre el aporte de las fundaciones empresariales de los sectores de minería e hidrocarburos a los ods relacionados con pobreza, hambre, salud, educación y género, que conforman el pilar personas de la agenda. Este estudio se realiza a partir de las temáticas atendidas por la inversión social privada (ISP) y su alineación con las metas e indicadores de los oDs; de la articulación con el gobierno y las entidades públicas de orden local, regional y nacional, y de las alianzas con organismos internacionales y el acceso a recursos de cooperación internacional.

Palabras clave: desarrollo sostenible; Agenda 2030 para el Desarrollo Sostenible; fundaciones; inversión social privada; alianzas; cooperación internacional.

* Profesional en Finanzas y Relaciones Internacionales. Candidata a maestría en Asuntos Internacionales de la Universidad Externado de Colombia. Bogotá (Colombia). Estudiante de la maestría en Gestión de riesgos en países del sur de Sciences Po. Bordeaux (Francia). [angela.munevar@est.uexternado.edu.co]; [https://orcid.org/00000002-7247-2360].

Trabajo desarrollado en el marco de la Maestría en Asuntos Internacionales de la Universidad Externado de Colombia, dirigido por el docente-investigador Oscar Iván Pérez Hoyos.

Recibido: 25 de junio de 2019 / Modificado: 30 de julio de 2019 / Aceptado: 20 de agosto de 2019

Para citar este artículo

Munévar, A. S. (2020). Las fundaciones empresariales en el cumplimiento de los oDs: una mirada desde los sectores de minería e hidrocarburos. OPERA, 26, 173-194.

DOI: https://doi.org/10.18601/16578651.n26.09 


\section{BUSINESS FOUNDATIONS IN ACCOMPLISHING SDGS: A VIEW FROM THE MINING AND HYDROCARBON SECTORS}

\section{Abstract}

The Sustainable Development agenda for 2030 requires action by diverse actors, like the government, the private sector and the social sector. This article analyses the contribution of corporate foundations of mining and hydrocarbon sectors in Colombia to the SDGs related to poverty, hunger, health, education and gender equality. In that sense, it becomes relevant to identify the thematic areas covered by Private Social Investment, its alignment with the established goals and targets; the articulation with the government and local, regional and national public entities; and finally, partnership with international organisms and the access to international cooperation resources.

Key words: Sustainable development; Agenda 2030; foundations; private social investment; partnership; international cooperation.

\section{INTRODUCCIÓN}

Los objetivos de desarrollo sostenible (oDs) constituyen la agenda del desarrollo establecida en la Organización de las Naciones Unidas (ONU), por medio de la cual 193 países -entre ellos Colombia- adoptaron una serie de compromisos relacionados con el crecimiento económico, la inclusión social y la sostenibilidad ambiental. La agenda estipula cinco esferas de importancia crítica: personas, prosperidad, planeta, paz y alianzas (PNUD, s. f.). Cada una de ellas reúne ciertos objetivos y metas, complementarios entre sí, que buscan contribuir al desarrollo sostenible.

A pesar del compromiso del Gobierno nacional de Colombia con el cumplimiento de la Agenda 2030 para el Desarrollo Sostenible (Agenda 2030), existen múltiples obstáculos que dificultan el cumplimiento de las ambiciosas metas. Por ejemplo, se plantea que para el año 2030 el Índice de Pobreza Multidimensional (IPM) de Colombia debe llegar al 8,4\%, no obstante, en 2018 este índice fue del 19,6\% (DANe, 2019). Por su parte, se prevé que la tasa de mortalidad materna en 2030 sea de 32 por cada 100.000 nacidos vivos, sin embargo, en 2017 esta cifra fue de 51 por cada 100.000 nacidos vivos (Comisión ods, 2019). Por último, se planteó que la tasa de mortalidad por desnutrición en niños (menores de 5 años) en 2030 debe ser de 5 por cada 100.000 niños, no obstante, en 2016 esta cifra fue de 8,24 (Ministerio de Salud y Protección Social, 2018, p. 134).

Adicionalmente, las lecciones aprendidas con la implementación de los objetivos de desarrollo del milenio (oDM), entre 2000 y 2015, señalan que el Estado colombiano por sí solo no cuenta con las capacidades suficientes, tanto en financiación como en cobertura, para alcanzar las metas propuestas. Los ODM se financiaron con recursos del Gobierno nacional que resultaron insuficientes por la falta de planeación financiera y el desconocimiento inicial de los costos totales de implementación (DNP, 2018b). 
Así las cosas, lograr las metas propuestas en la Agenda 2030 requiere de un esfuerzo importante, no solamente del Gobierno nacional, sino también de todos los actores de la sociedad que han sido llamados a participar y a crear una discusión colectiva sobre su implementación. Por tanto, la ejecución de los oDs a partir de 2015 requiere la incorporación de recursos adicionales provenientes de empresas, fundaciones, de la banca multilateral y de cooperación internacional, de acuerdo con lo estipulado por el CONPES 3918 de 2018 (DNP, 2018a, p. 19). No obstante, la participación de empresas y fundaciones empresariales requiere de un diálogo cercano con el Gobierno y de una rigurosa alineación institucional que permita la creación de alianzas y estrategias de colaboración incluyentes.

El objetivo de la investigación es analizar la articulación de las fundaciones empresariales de los sectores de minería e hidrocarburos que hacen parte de la Asociación de Fundaciones Petroleras (AFP) ${ }^{1}$ y de la Asociación de Fundaciones Familiares y Empresariales (AFE), con los cinco objetivos que conforman el pilarpersonas de la Agenda 2030 en Colombia, a partir del diálogo y la articulación con el Gobierno en sus distintos niveles.

El pilar personas de los oDs está conformado por los siguientes objetivos: erradicación de la pobreza (objetivo 1), hambre cero (objetivo 2), salud y bienestar (objetivo 3), educación de calidad (objetivo 4) e igualdad de género (objetivo 5). El estudio de este pilar se debe a que las temáticas que abordan constituyen el principal enfoque de las fundaciones empresariales que trabajan para mejorar las condiciones de vida de la población radicada en sus zonas de influencia, a partir de una perspectiva económica y social (Villar, 2018).

La AFP reúne las cinco principales fundaciones petroleras de Colombia y se encarga de representar y promover sus intereses, de difundir su trabajo y contribuir al desarrollo local. Por su parte, la AFE reúne 72 fundaciones colombianas, de las cuales 10 pertenecen a los sectores de minería e hidrocarburos. Esta asociación tiene como objetivo visibilizar las acciones de las fundaciones, lograr una mayor articulación entre estas y grupos de interés e incidir en la agenda pública (AFE, 2018).

El énfasis en los sectores de minería e hidrocarburos se debe a que estos representan sectores estratégicos de la economía nacional, como una de las principales fuentes de divisas y de ingresos del Estado, así como por la sólida estructura de responsabilidad social empresarial (RSE) que han desarrollado sus empresas en las zonas de influencia directa en Colombia, con el fin de generar valor en las comunidades donde tienen influencia, contrarrestar los efectos sociales y ambientales, los sentimientos públicos negativos y construir un "capital de reputación" (Cassand, 2014).

Los sectores de minería e hidrocarburos han contribuido al crecimiento del producto interno bruto (PIB) per cápita del país (Malagón, 2016); no obstante, este crecimiento será insuficiente si no se refleja en la mejora en las condiciones de vida de la población, pues no

1 Se encuentra en proceso de liquidación desde febrero de 2019. 
estaría contribuyendo al bienestar y desarrollo del país. Más aún, teniendo en cuenta que la explotación minero-energética ha ocasionado cambios sociales y ambientales en las regiones donde se lleva a cabo (Rudas y Espitia, 2013).

Los objetivos específicos de la investigación son: a) identificar las temáticas atendidas por la inversión social privada (ISP) de las fundaciones de los sectores de minería e hidrocarburos y su relación con las metas e indicadores de la Agenda 2030, b) establecer el tipo de alianzas que priorizan las fundaciones empresariales de los sectores de minería e hidrocarburos, y c) determinar el rol de la cooperación internacional en la articulación de las fundaciones empresariales de AFP y AFE con los oDs.

El artículo responde a la pregunta: ¿de qué forma los programas realizados por fundaciones empresariales de los sectores de minería e hidrocarburos se articulan con los ods que conforman el pilar personas en las zonas en las que operan en Colombia? Para ello, se parte de la siguiente hipótesis: las fundaciones empresariales de los sectores de minería e hidrocarburos se articulan con los ods que conforman el pilar personas en la medida en que las áreas temáticas a las cuales se dirige su inversión social privada concuerdan con aquellas priorizadas por los oDs que conforman dicho pilar; asimismo, se articulan al crear alianzas con otros actores, principalmente del sector público, pero también del sector privado y de la sociedad civil, y al establecer relaciones de cooperación con organizaciones internacionales.

El artículo está estructurado de la siguiente manera: en primer lugar, se realiza una aproximación teórica al desarrollo sostenible y se exponen tres categorías de investigación: las temáticas atendidas por la inversión social privada de las fundaciones empresariales, la relación entre las fundaciones empresariales y el Gobierno en sus distintos niveles y el aporte de la cooperación internacional en el desarrollo sostenible. Enseguida, se exponen los principales hallazgos de la contribución de las fundaciones empresariales de los sectores de minería e hidrocarburos al cumplimiento de los oDs de acuerdo con las categorías de investigación. Finalmente, se exponen las principales conclusiones de la investigación.

\section{EL DESARROLLO SOSTENIBLE: APROXIMACIÓN TEÓRICA}

El marco teórico empleado durante la investigación gira en torno a la teoría de desarrollo sostenible como fundamento de los oDs.

El desarrollo sostenible se describe como un proceso complejo al combinar los términos desarrollo, que expresa un cambio progresivo, y sostenible, que indica un mantenimiento en el tiempo (Gallopín, 2010, citado por Mora y Martínez, 2018, p. 31). En esta misma línea, Gallopín (2003) plantea una diferencia importante entre los términos sostenibilidad y desarrollo sostenible, en cuanto el primero se aplica al mantenimiento del estado de un sistema, mientras que el segundo suscita la idea de un cambio gradual y direccional, hacia el mejoramiento de la condición humana y el despliegue de potencialidades.

El concepto de desarrollo sostenible se formalizó por primera vez en el informe de la Comisión sobre el Medio Ambiente y el Desa- 
rrollo de las Naciones Unidas, conocida como Comisión de Brudtland de 1987. Originalmente, el concepto de desarrollo sostenible se planteó como "aquel que satisface las necesidades del presente sin comprometer la capacidad de las futuras generaciones para satisfacer las propias" (Leal, 2013, citado por Salas, 2017). Esta definición se construyó a partir de una perspectiva intergeneracional, haciendo énfasis en la compensación a las generaciones futuras por el uso de los recursos de las actuales generaciones, y en general en la satisfacción de las necesidades de las generaciones presentes y futuras (Salas, 2017).

Con el tiempo, este concepto evolucionó hacia un enfoque más holístico que comprende la interacción entre tres sistemas complejos: la economía mundial, la sociedad global y el medio ambiente físico de la tierra. De acuerdo con Sachs (2014), el desarrollo sostenible pretende construir un mundo donde el progreso económico sea un proceso generalizado, donde se elimine la pobreza extrema, se reduzcan las desigualdades y el medio ambiente esté protegido frente a degradaciones inducidas por la actividad humana.

No obstante, el desarrollo sostenible requiere de un cuarto componente: la buena gobernanza (Sachs, 2014). La noción de "buena gobernanza" se basa en los principios de responsabilidad, transparencia y participación (Sachs, 2014, citado por Ruiz, 2017, p. 159) y se refiere, por una parte, a los gobiernos en sus distintos niveles, a partir de la prestación de servicios básicos como sanidad, educación, la construcción de infraestructura, el desarrollo de tecnologías innovadoras, el control del crimen, la violencia y la corrupción, así como la reglamentación para la protección del medio ambiente. Adicionalmente, hace referencia a la actuación de las empresas, en la medida en que estas pueden influir en el bienestar social, el respeto por las reglamentaciones fiscales y la protección del medio ambiente.

Una de las premisas del desarrollo sostenible es la interacción de sistemas complejos, que hace referencia a la complejidad de la interacción de los sistemas económico, social, ambiental y de gobernanza (Sachs, 2014). Esta premisa reconoce la existencia de características o resultados imprevistos frente a los cambios que se puedan generar en alguno de los componentes del sistema y que afectan el funcionamiento de este en su conjunto. Bajo esta lógica, fenómenos tan complejos como la pobreza y la desigualdad son el resultado de múltiples factores presentes en el sistema y, por tanto, es erróneo tratar de encontrar una solución única. La interacción de los sistemas sugiere la adopción de múltiples medidas y actores para hacer frente a estos fenómenos (Gallopín, 2003).

El enfoque normativo del desarrollo sostenible realiza una serie de recomendaciones u objetivos sobre el deber ser de la sociedad, de manera que se conforman como una guía para construir una buena sociedad, en el sentido de promover la prosperidad económica, la inclusión y cohesión social, la sostenibilidad ambiental y la buena gobernanza (Sachs, 2014).

Desde esta perspectiva, el desarrollo de una sociedad no se limita, entonces, al incremento del nivel de ingresos, midiendo el nivel de riqueza en términos de PIB per cápita (Salas, 2017), sino que tiene en cuenta la distribución de dicha riqueza y la desigualdad existente, as- 
pectos que reflejan de manera más precisa las condiciones sociales y la distribución de bienestar de una población. Una buena sociedad también tiene una buena gestión ambiental, a fin de garantizar la sostenibilidad de los recursos naturales y la biodiversidad, así como una buena gobernanza que produce un sentimiento de bienestar y seguridad (Sachs, 2014).

En este mismo sentido, Gallopín (2003) sostiene que las constantes transformaciones demográficas, tecnológicas, económicas y ambientales ponen de manifiesto que el desarrollo sostenible debe orientarse, no solo a preservar la base ecológica y la habitabilidad, sino también a aumentar las capacidades para enfrentar los cambios, conservando y ampliando los recursos disponibles.

En definitiva, los oDs son una agenda internacional para el cambio que surge como resultado de la noción holística de desarrollo sostenible; de ahí que promueven un "crecimiento económico socialmente inclusivo y ambientalmente sostenible" (Sachs, 2014 p. $11)$.

\section{Categorías de análisis}

Con el objetivo de evaluar los aportes de las fundaciones empresariales de los sectores de minería e hidrocarburos al cumplimiento de los ons que conforman el pilar personas de la Agenda 2030, se seleccionan tres categorías de análisis, a saber: las temáticas atendidas por la ISP de las fundaciones empresariales, la relación entre las fundaciones empresariales y el Gobierno en sus distintos niveles y el papel de la cooperación internacional en el desarrollo de proyectos de las fundaciones y su articulación con los oDs.

\section{Temáticas atendidas por la inversión social privada}

Las líneas de acción o las temáticas atendidas por la ISP varían de acuerdo con los ejes estratégicos de las fundaciones empresariales y las empresas. En Colombia, las líneas de acción más recurrentes en el quehacer de las fundaciones son educación (70\%); generación de ingresos (44\%); desarrollo de base y trabajo con comunidades (41\%); paz, superación del conflicto y derechos humanos $(25,4 \%)$; arte, cultura y deporte $(24,7 \%)$; cultura y convivencia ciudadana (20,3\%); salud (20,3\%); vivienda $(18,9 \%)$; protección del medio ambiente (15\%); discapacidad y adultos mayores $(13,5 \%)$; nutrición y seguridad alimentaria (12\%) y movilidad sostenible $(1,7 \%)$ (Villar, 2015, p. 73).

Las fundaciones empresariales pueden contribuir al cumplimiento de los oDs que conforman el pilar personas a partir, por ejemplo, de proyectos y programas educativos, de fortalecimiento de capacidades, de generación de ingresos y desarrollo productivo, y de salud, vivienda y seguridad alimentaria, que busquen la provisión de servicios de calidad y mejoren las condiciones de vida de las poblaciones beneficiarias.

En primer lugar, las estrategias de generación de capacidades son, de acuerdo con la Fundación DIs y la Fundación Promigas (2012), un medio para "asegurar la apropiación y comprensión por parte de las comunidades, 
de su papel y responsabilidad frente a su propio desarrollo" (p. 44); asimismo permiten que "los procesos sociales sean sostenibles al terminar la intervención y, consecuentemente, permiten eliminar la dependencia de los programas de la fundación y de la empresa” (p. 44). Por su parte, los proyectos productivos de generación de ingresos o programas de emprendimiento contribuyen a reducir la pobreza y a promover el desarrollo humano, técnico y empresarial. En este ámbito, la contribución de las fundaciones se puede dar a partir de asesorías técnicas, acceso a recursos financieros y generación de capacidades colectivas (OCDE, 2016). De otro lado, los programas educativos y de formación de jóvenes tienen un gran potencial para generar igualdad de oportunidades y para aportar al cierre de brechas de ingresos o de género. Estos programas contribuyen al avance de indicadores sociales como, por ejemplo, el índice de desarrollo humano (IDH) ${ }^{2}$, al ser una medida que combina la esperanza de vida, el ingreso y el nivel educativo de las personas (Villar, 2015).

Adicional a las temáticas abordadas por la ISP, es deseable que la contribución de las fundaciones se relacione directamente con las metas e indicadores que fueron establecidos por el Gobierno nacional para el cumplimiento de cada uno de los 17 objetivos. Así, por ejemplo, los programas cuyo fin sea la reducción de la pobreza, deberían ser formulados con base en indicadores como el Índice de pobreza multidimensional o el Índice de pobreza monetaria. Los programas de nutrición y seguridad alimentaria deberían contribuir a la disminución de la tasa de mortalidad por desnutrición en niños menores de 5 años o de la proporción de menores de 6 meses con lactancia materna exclusiva, y así sucesivamente.

De acuerdo con el principio de transversalidad e interdependencia de los objetivos, un programa o proyecto puede movilizar varios oDs simultáneamente y, de esta manera, contribuir al cumplimiento de las metas de más de un objetivo (Comisión ods, 2018). Así, por ejemplo, un programa educativo que incremente el acceso de mujeres a educación técnica o profesional contribuye al cumplimiento de indicadores como cobertura en educación superior (oDs 4) y a disminuir la brecha de ingreso entre hombres y mujeres (ODS 5).

A pesar de los aportes de la isp, cabe resaltar que por la naturaleza de los actores, es decir, las fundaciones empresariales, las acciones realizadas a través de inversión social privada corresponden a gestiones concretas en las zonas de influencia de las empresas (Villar, 2018 , p. 48). Por tanto, el sector privado es considerado un socio de desarrollo, que juega un papel complementario, pero el Estado es y seguirá siendo el actor fundamental que lidera las políticas de desarrollo económico (PintorPirzkall, 2017).

\footnotetext{
2 Índice elaborado por el Programa de las Naciones Unidas para el Desarrollo (PNUD) que mide el desarrollo humano de cada país.
} 


\section{Relación entre las fundaciones empresariales y el Gobierno en sus distintos niveles}

Las fundaciones empresariales contribuyen al cumplimiento de los ons en Colombia en la medida en que exista una alineación de su gestión social con la hoja de ruta que ha planteado el Gobierno como estrategia de implementación, para lo cual se requiere de un diálogo constante y articulación de programas a través de alianzas con entidades gubernamentales en sus distintos niveles.

El diálogo entre fundaciones, entes gubernamentales y otros actores del sector social es una herramienta valiosa para alcanzar una mejor colaboración, ya que permite un conocimiento mutuo de los actores, así como tener claridad y precisión acerca de los objetivos institucionales y, a partir de ello, se pueden unir intereses e impulsar la creación de espacios de cooperación (OCDE netFWD, 2016).

La disposición de espacios y mecanismos de diálogo es clave, ya sea a través de ámbitos que han sido institucionalizados (como foros o comités) o de negociaciones que surgen de manera coyuntural (OCDE netFwd, 2016). No obstante, la posibilidad de acercamiento de las fundaciones con el Gobierno depende del sistema político y de la concepción que se tenga del papel del sector privado en torno a temas sociales que tradicionalmente han sido de manejo del sector público (Villar, 2015).

La cooperación entre las fundaciones empresariales y el Gobierno nacional y local es fundamental para generar un mayor impacto de la actividad filantrópica y la Isp. En el caso colombiano, las fundaciones se caracterizan por su interés en establecer relaciones de colaboración, la mayor parte de ellas colabora con otras fundaciones, al igual que con el gobierno estableciendo alianzas público-privadas (APP) y cofinanciando proyectos (Villar, 2018).

En este sentido, la Red Global de Fundaciones Trabajando para el Desarrollo de la OCDE - netFWD estipula que la construcción de alianzas entre fundaciones y otros actores se puede llevar a cabo en las distintas etapas de los programas ejecutados por las fundaciones, como en el diseño, en la implementación o en la evaluación. Si bien en general existe una valoración positiva de las alianzas para el desarrollo, las directrices de la netFwD hacen énfasis en características como la duración, la formalidad y la naturaleza de los recursos. Asimismo, la construcción de alianzas puede enfrentar obstáculos como tramitología compleja y controles que encarecen los costos de operación, así como la dependencia en los periodos políticos (OCDE netFwD, 2016).

Stott (2017) identifica una serie de incentivos para el Gobierno, el sector privado, los donantes y otros actores al momento de construir alianzas. Entre estos se destaca que las alianzas aseguran un mayor cubrimiento de servicios, mejoran la reputación, la legitimidad y la credibilidad, garantizan nuevas fuentes de financiamiento, mejoran el acceso a la información, incentivan la innovación y promueven programas y proyectos de largo plazo. Esta autora afirma que el éxito de las alianzas depende del buen entendimiento de los incentivos de trabajar en equipo, lo cual puede mejorar el potencial de las acciones colaborativas para contribuir más ampliamente a la Agenda 2030. 
Este tema es abordado en la misma Agenda 2030 a partir del ods 17, "Alianzas para lograr los objetivos", según el cual el conjunto de oDs solo se puede lograr con el compromiso a favor de alianzas globales y la cooperación entre los diferentes niveles de gobierno, la sociedad civil, las comunidades y otros actores (Comisión ods, 2018).

\section{Aporte de la cooperación internacional al desarrollo sostenible}

Pauselli (2013) explica la cooperación internacional como instrumento para generar desarrollo a partir de las diferentes teorías de relaciones internacionales. En este proceso, el autor identifica una explicación humanitaria desde el constructivismo, en contraste con una explicación más egoísta a partir de teorías como el realismo y el marxismo.

Para fines de la presente investigación, se profundiza en el enfoque dado por el constructivismo, el cual se centra en la influencia de las ideas y de las normas en el comportamiento de los Estados y en su capacidad de cambio, lo que permite pasar de sistemas competitivos a sistemas de cooperación (Pauselli, 2013). Según el constructivismo, las identidades estatales determinan quién es cada Estado y cuáles son sus intereses, y propone la noción de identidad colectiva donde el interés propio y el interés colectivo surgen de las relaciones entre Estados y su proceso de identificación (Wendt 1994, citado por Pauselli, 2013).

La explicación humanitaria de la ayuda internacional se relaciona con el proceso de identificación positiva que tienen los donantes con las sociedades de los Estados receptores.
De esta manera, la cooperación al desarrollo se puede entender como la evolución de la identidad colectiva mundial, en la cual la norma internacional presiona a los Estados ricos a ayudar a los Estados pobres para mejorar sus condiciones (Pauselli, 2013, p. 87). Adicional a esto, Ayllón (2007) argumenta la cooperación internacional desde el constructivismo como una obligación imperfecta de provisión de las necesidades básicas (argumento de carácter deontológico) y como una respuesta ética a los problemas que pueden remediarse con la capacidad técnica de los donantes (argumento de carácter utilitario).

Para abordar el papel de la cooperación internacional en el aporte de las fundaciones empresariales al cumplimiento de los ods en Colombia, se debe entender que el sector privado puede actuar como cooperante o como receptor de recursos de cooperación internacional. De acuerdo con APC Colombia y Fundación ANDI, el sector privado "actúa como cooperante a través de sus donaciones o ISP, cuando estas traspasan fronteras, y como receptor, directamente desde la empresa o a través de las fundaciones empresariales" (2016, p. 11).

Bajo esta perspectiva, las empresas y las fundaciones empresariales, como actores no oficiales del sistema de cooperación internacional, pueden contribuir al desarrollo sostenible en la medida en que se dé una articulación entre los recursos de cooperación internacional y la ISP, para dar lugar al fortalecimiento de agendas de desarrollo compartidas y proyectos sociales que contribuyan a la construcción de una sociedad más equitativa (APC Colombia y Fundación ANDI, 2016). 
La cooperación internacional proveniente de las donaciones de países, gobiernos locales y entidades no gubernamentales; al igual que los recursos provenientes de iniciativas del sector privado, tanto de empresas como de fundaciones, son concebidos como una fuente de recursos dentro del esquema de financiamiento de los ops en Colombia (Comisión ods, 2018). Así, los recursos de la cooperación internacional pueden contribuir a mejorar el acceso al crédito, a promover la innovación en los emprendimientos y proyectos productivos, a desarrollar tecnología y brindar formación técnica (García y Salcedo, 2017).

\section{METODOLOGÍA}

Para el desarrollo de la investigación se selecciona una muestra típica de 10 fundaciones empresariales de los sectores de minería e hidrocarburos. Esta es una forma de "muestra homogénea donde se eligen casos de un perfil similar, pero que se consideran representativos de un segmento de la población" (Hernández, 2010, p. 398). En este caso, las fundaciones seleccionadas están asociadas a las empresas más importantes de dichos sectores en los distintos niveles de la cadena productiva ${ }^{3}$ y tienen presencia en las principales regiones del país donde se realizan actividades de extracción, producción y comercialización de minerales e hidrocarburos. Las fundaciones fueron seleccionadas a partir de criterios como: 1) hacer parte de una o varias empresas de los sectores de minería e hidrocarburos en Colombia, 2) ser miembro de AFP o de la AFE, y 3) presentar memorias de sostenibilidad e informes de gestión accesibles al público.

De las diez fundaciones seleccionadas, cinco hacen parte de la (AFP) y cinco hacen parte de la (AFE). Las fundaciones que forman parte de AFP son: Fundación Ecopetrol para el Desarrollo del Magdalena Medio (Fundesmag), Fundación Alto Magdalena (FAM), Fundación Amanecer, Fundación Oleoductos de Colombia (FODC) y Fundación Alcaraván. Por su parte, las fundaciones que hacen parte de la AFE son: Fundación Cerrejón, Fundación Terpel, Fundación Social Acerías Paz del Río, Fundación Surtigas y Fundación Gases del Caribe (tabla 1).

El espacio de investigación abarca los departamentos en los que las fundaciones empresariales mencionadas adelantan sus acciones, es decir, Antioquia (2 fundaciones), Bolívar (3), Boyacá (5), Cesar (3), Córdoba (3), Cundinamarca (3), Santander (3), La Guajira (2), Arauca (3), Casanare (3) y Meta (3). Se toman varias regiones teniendo en cuenta que algunos programas de dichas fundaciones se ejecutan en varios territorios, según la zona de influencia y los grupos de interés establecidos. El periodo de investigación va de 2015 -año de inicio de la implementación de los oDs- hasta 2018 -el año más reciente del cual se tiene información consolidada-.

\footnotetext{
3 La cadena productiva de los sectores de minería e hidrocarburos corresponde al conjunto de actividades relacionadas con la exploración, la producción, el transporte, la refinación o procesamiento y la comercialización de recursos naturales no renovables como petróleo, gas, carbón y minerales.
} 
TABLA 1. FUNDACIONES EMPRESARIALES DE LOS SECTORES DE MINERÍA E HIDROCARBUROS

\begin{tabular}{|c|c|c|c|}
\hline Asociación & Empresa (s) & $\begin{array}{l}\text { Actividad de la(s) } \\
\text { empresa(s) }\end{array}$ & $\begin{array}{l}\text { Departamentos de } \\
\text { intervención }\end{array}$ \\
\hline \multicolumn{4}{|c|}{ Asociación de Fundaciones Petroleras } \\
\hline $\begin{array}{l}\text { Fundación Ecopetrol para el } \\
\text { Desarrollo del Magdalena } \\
\text { Medio (FUNDESMAG) }\end{array}$ & Ecopetrol S.A. & $\begin{array}{l}\text { Exploración y producción } \\
\text { de hidrocarburos }\end{array}$ & $\begin{array}{l}\text { Magdalena medio: } \\
\text { Cesar, Bolívar, San- } \\
\text { tander }\end{array}$ \\
\hline $\begin{array}{l}\text { Fundación Alto Magdalena } \\
\text { (FAM) }\end{array}$ & $\begin{array}{l}\text { Ecopetrol S.A. } \\
\text { Hocol S.A. }\end{array}$ & $\begin{array}{l}\text { Exploración y producción } \\
\text { de hidrocarburos }\end{array}$ & $\begin{array}{l}\text { Huila, Caquetá, } \\
\text { Putumayo y Meta }\end{array}$ \\
\hline Fundación Amanecer & $\begin{array}{l}\text { Ecopetrol S.A. / Equion Ener- } \\
\text { gía Ltd. / Santiago Oil Com- } \\
\text { pany / Emerald Energy }\end{array}$ & $\begin{array}{l}\text { Exploración y producción } \\
\text { de hidrocarburos }\end{array}$ & $\begin{array}{l}\text { Casanare, Meta, } \\
\text { Cundinamarca, Arauca } \\
\text { y Boyacá }\end{array}$ \\
\hline $\begin{array}{l}\text { Fundación Oleoductos de } \\
\text { Colombia (FODC) }\end{array}$ & $\begin{array}{l}\text { Oleoducto de Colombia S.A. } \\
\text { (ODC) / Oleoducto Central S.A. } \\
\text { (OCENSA) }\end{array}$ & $\begin{array}{l}\text { Transporte de hidrocar- } \\
\text { buros }\end{array}$ & $\begin{array}{l}\text { Casanare, Santander, } \\
\text { Boyacá, Antioquia, } \\
\text { Córdoba y Sucre }\end{array}$ \\
\hline Fundación Alcaraván & $\begin{array}{l}\text { Ecopetrol S.A. / Occidental de } \\
\text { Colombia (oxy) }\end{array}$ & $\begin{array}{l}\text { Exploración y producción } \\
\text { de hidrocarburos }\end{array}$ & Arauca y Boyacá \\
\hline \multicolumn{4}{|c|}{ Asociación de Fundaciones Familiares y Empresariales } \\
\hline Fundación Cerrejón & El Cerrejón & $\begin{array}{l}\text { Extracción y explotación de } \\
\text { carbón }\end{array}$ & La Guajira \\
\hline Fundación Terpel & Terpel & $\begin{array}{l}\text { Distribuidora y comerciali- } \\
\text { zadora de combustibles y } \\
\text { lubricantes }\end{array}$ & $\begin{array}{l}\text { Antioquia, Bolívar, } \\
\text { Boyacá, Cesar, Cór- } \\
\text { doba, Cundinamarca, } \\
\text { Santander, La Guajira, } \\
\text { Arauca, Casanare, y } \\
\text { Meta. Total: } 28 \text { depar- } \\
\text { tamentos }\end{array}$ \\
\hline $\begin{array}{l}\text { Fundación Social Acerías Paz } \\
\text { del Río }\end{array}$ & Acerías Paz del Río & Producción de acero & $\begin{array}{l}\text { Cundinamarca y } \\
\text { Boyacá }\end{array}$ \\
\hline Fundación Surtigas & Surtigas & $\begin{array}{l}\text { Distribuidora y comerciali- } \\
\text { zadora de gas }\end{array}$ & $\begin{array}{l}\text { Bolívar, Córdoba y } \\
\text { Sucre }\end{array}$ \\
\hline Fundación Gases del Caribe & Gases del Caribe & $\begin{array}{l}\text { Distribuidora y comerciali- } \\
\text { zadora de gas natural }\end{array}$ & $\begin{array}{l}\text { Atlántico, Magdalena } \\
\text { y Cesar }\end{array}$ \\
\hline
\end{tabular}

Fuente: elaboración propia con base en informes de sostenibilidad y páginas web de las fundaciones.

La presente investigación es de tipo cualitativo y el estudio se lleva a cabo mediante revisión documental de distintos tipos de fuentes primarias y secundarias, a saber: textos normativos, políticas públicas (Consejo Nacional de Política Económica y Social - CONPES), informes técnicos, memorias de sostenibilidad, páginas web y artículos de prensa. Las principales organizaciones consultadas son el Departamento Nacional de Planeación (DNP), Plataforma de Filantropía para los ods-Colombia, la AFE y la AFP.

La revisión documental se hace con el fin de establecer el conocimiento y el dominio de las fundaciones seleccionadas sobre los oDs priorizados en el estudio, la existencia de 
alianzas con los gobiernos locales y organismos internacionales, los métodos de medición de resultados y su contribución con las metas y los indicadores de la Agenda 2030. A partir de ello se extraen datos y estadísticas que son recolectados y estructurados en una matriz de información y son utilizados como insumo para la investigación. La información recolectada se contrasta con una encuesta online realizada a las fundaciones que hacen parte de la muestra.

La encuesta es elaborada con base en las categorías de análisis antes mencionadas. El cuestionario utilizado para la realización de la encuesta cuenta con once preguntas, de las cuales nueve son de selección múltiple no excluyente y dos son preguntas abiertas. La encuesta es diligenciada por empleados de las fundaciones de nivel directivo que tienen conocimiento sobre los ons y la gestión realizada por la fundación.

\section{EL APORTE DE LAS FUNDACIONES EMPRESARIALES AL CUMPLIMIENTO DE LOS ODS SOCIALES: EVIDENCIA EMPÍRICA}

\section{Temáticas abordadas por la inversión social} privada de las fundaciones empresariales de los sectores de minería e hidrocarburos

De acuerdo con los informes de sostenibilidad de las fundaciones empresariales se evidencia que sus programas se desarrollan dentro de una dimensión social, económica y ambiental, ámbitos que hacen parte de la Agenda 2030. Esta información se obtuvo como resultado de una matriz de información donde se recopilaron las líneas de acción o ejes estratégicos establecidos por las fundaciones, de la cual se destaca que de las diez fundaciones, seis trabajan en pro del desarrollo empresarial y productivo, cinco de ellas establecieron la educación como eje estratégico, cuatro fundaciones priorizan el desarrollo humano y el fortalecimiento de capacidades y cuatro fundaciones establecieron el desarrollo y la sostenibilidad ambiental como asunto prioritario.

Al relacionar los ejes estratégicos y programas realizados por las fundaciones con las temáticas de los oDs, particularmente los relacionados con el eje de personas de la Agenda 2030, se observa, en primer lugar, que nueve de diez fundaciones llevan a cabo proyectos que tienen como finalidad la erradicación de la pobreza (Objetivo 1). Para ello, desarrollan programas de acceso a servicios básicos, construcción y mejoramiento de vivienda y desarrollo de proyectos productivos a partir del fortalecimiento de capacidades (capacitaciones) y microcréditos. Segundo, nueve de diez fundaciones llevan a cabo programas y proyectos educativos (Objetivo 4), a partir de becas educativas, programas de alfabetización, planes de apoyo educativos, educación en tecnologías de la información, derechos humanos, liderazgo, entre otros. Tercero, seis de diez fundaciones implementaron programas de igualdad de género (Objetivo 5) ya sea de manera transversal o a partir de programas específicos. Cuarto, en lo relacionado con erradicación del hambre (Objetivo 2), dos fundaciones desarrollan proyectos específicos de seguridad alimentaria. Finalmente, una fundación trabaja en salud y bienestar a través de la implementación de brigadas de salud (figura 1). 
FIGURA 1. NÚMERO DE FUNDACIONES ALINEADAS CON LAS TEMÁTICAS DE LOS ODS

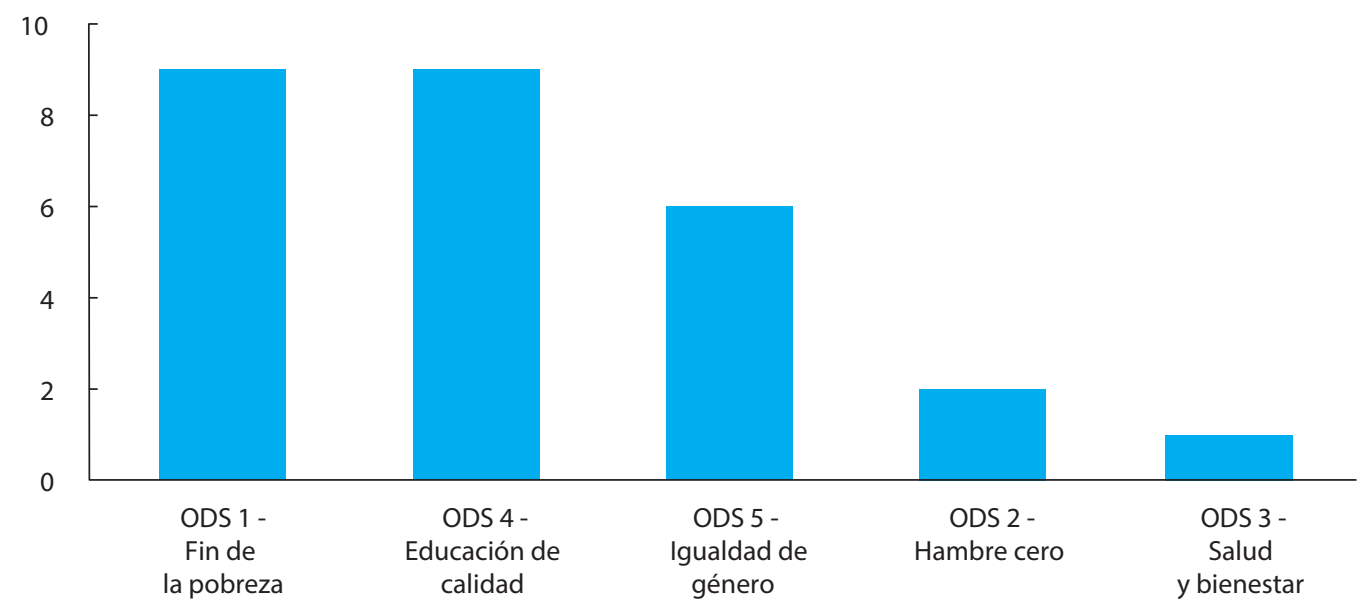

Fuente: elaboración propia con base en informes de sostenibilidad, páginas web y encuesta realizada a las fundaciones.

En cuanto a la población beneficiaria de los programas de las fundaciones de los sectores de minería e hidrocarburos, se evidencia que cuatro de las diez fundaciones trabajan principalmente con mujeres (FODC, Fundación Amanecer, Fundación Alcaraván y Fundesmag) y tres lo hacen principalmente con jóvenes (Fundación Terpel, Fundación Acerías Paz del Río y Fundación Surtigas). Las demás fundaciones trabajan con la comunidad local, es decir, la radicada en sus zonas de influencia directa.

Estos datos, junto a la encuesta realizada, confirman que los temas priorizados por las fundaciones coinciden con los objetivos que conforman la dimensión social o el pilar personas de la Agenda 2030. Sin embargo, esto no implica que sus programas se hayan formulado e implementado en el marco de dicha Agenda. En general, a partir del análisis de los informes de sostenibilidad, se infiere que existe un cono- cimiento de esta por parte de las fundaciones de los sectores de minería e hidrocarburos, nueve de las diez fundaciones mencionan los oDs en los informes de sostenibilidad o gestión, ya sea de manera general, mencionando su compromiso con la Agenda 2030 sostenible o de manera específica, señalando las temáticas de los ods que prioriza.

Sumado a lo anterior, y como se mencionó en la primera parte del documento, es deseable que la gestión de las fundaciones en favor de los ODS se mida según la incidencia en las metas e indicadores establecidos por el Gobierno nacional para este fin. En los informes de sostenibilidad de las fundaciones se observa que el resultado de los programas es medido de acuerdo con el número de beneficiaros y relacionado de manera general con la reducción de la pobreza y de las desigualdades en las zonas de influencia. Sin embargo, los resultados de su 
gestión no han sido relacionados directamente con los indicadores y las metas de los oDs.

Aunque determinar la contribución de las fundaciones y del sector privado en general en la Agenda 2030 no es un proceso sencillo, debido a la multiplicidad de actores y la falta de un método estandarizado de reporte de resultados (DNP, 2018), el DNP sugiere la adopción de los estándares de la Global Reporting Initiative (GRI) por parte de las empresas y las fundaciones empresariales, al ser un mecanismo uniforme que permite medir la contribución y el impacto de los actores privados de una manera efectiva y comparable. Estos estándares son los más utilizados en la elaboración de informes de sostenibilidad por las compañías a nivel internacional (DNP, 2018).

A pesar de ello, solamente dos de las diez fundaciones han elaborado los informes de sostenibilidad de conformidad con los estándares GRI (Fundación Amanecer y Fundesmag). En contraste, se evidencia que en los informes de las empresas es más recurrente el uso de esta metodología, como es el caso de Ocensa, Hocol, Cerrejón, Terpel y Surtigas. Estas son las mismas organizaciones que hacen parte de Pacto Global Colombia.

En definitiva, el trabajo de las fundaciones de los sectores de minería e hidrocarburos responde a las temáticas abordadas en el ámbito social de los ods. Ahora bien, el Gobierno nacional ha establecido metas e indicadores para medir los avances en materia de desarrollo sostenible a lo largo de su implementación, por lo tanto, es deseable que las empresas y fundaciones empresariales actúen de manera articulada a estos lineamientos y midan su contribución en los mismos términos, de manera que la información reportada sea homogénea, comparable y permita visibilizar la labor de las fundaciones en asuntos de desarrollo sostenible.

\section{¿Qué tipo de alianzas priorizan las fundaciones?}

Uno de los aspectos principales de la contribución de las fundaciones al cumplimiento de los ods se da a partir de la articulación y el diálogo con el Gobierno a nivel nacional, regional y local. Para una mayor articulación es necesario alinear la gestión social de las fundaciones con la hoja de ruta que ha planteado el Gobierno como estrategia de implementación de los ODS a partir del CONPES 3918 de 2018 y con los Planes de Desarrollo Territorial.

La interlocución y el diálogo entre las fundaciones empresariales de minería e hidrocarburos y las entidades del Gobierno en sus distintos niveles se da principalmente de manera directa en reuniones individuales, a través de comités donde participan múltiples actores y por medio de organizaciones como la AFE y la AFP. De acuerdo con las fundaciones encuestadas, estas dos organizaciones han contribuido a la articulación de las fundaciones con los oDs en la medida en que promueven el intercambio de conocimientos y experiencias, gestionan información de interés relacionada con los ODS, gestionan espacios de diálogo con otras entidades y han articulado agendas con aquellas entidades que se interesan por las mismas líneas de acción de las fundaciones.

Tanto la AFE como la AFP se encargan de visibilizar las acciones y de representar los intereses de las fundaciones para lograr una ma- 
yor articulación con sus grupos de interés. De acuerdo con la información recolectada, ambas asociaciones han trabajado con las fundaciones del sector para que estas incluyan dentro de sus planes de acción las temáticas de los oDs. Por su parte, la AFE ha tenido una agenda de relacionamiento con la Agencia Presidencial de Cooperación Internacional - APC Colombia, con el objetivo de mejorar la articulación entre la ISP y la cooperación internacional a partir de iniciativas como la documentación y publicación de experiencias exitosas de las fundaciones que puedan ser compartidas a nivel internacional (Montoya, 2018).

De acuerdo con los informes de sostenibilidad y la encuesta realizada, la totalidad de las fundaciones que hacen parte de la muestra reconocen la importancia de establecer vínculos y alianzas estratégicas con entidades gubernamentales y con otras entidades. El propósito o aporte es principalmente ampliar el conocimiento y hacer uso de la experiencia de los aliados (100\%), seguido de alcanzar una mayor cobertura y capacidad de implementación (80\%). Otros aspectos valorados, pero menos recurrentes, son la provisión de productos y servicios $(20 \%)$ y la posibilidad de cofinanciar proyectos $(20 \%)$.

Según lo establecido en los informes de sostenibilidad, las páginas web y la encuesta realizada, se realiza un listado de las alianzas establecidas por las fundaciones y se clasifican de acuerdo con el tipo de actor (tabla 2). De acuerdo con esta información, las diez fundaciones han llevado a cabo alianzas con gobiernos locales, regionales y entidades del sector público en general, y ocho de ellas las priorizan frente a alianzas con otro tipo de actores (tabla
2). Las alianzas con el sector privado ocupan el segundo lugar en frecuencia, seguidas de alianzas con la academia. En contraste, las alianzas con organismos internacionales son las menos recurrentes.

De acuerdo con la temática de los programas, estas fundaciones mantienen alianzas con entidades como APC Colombia, el Ministerio de Educación, el Ministerio de Ambiente, Ministerio de Comercio, Industria y Turismo, y Cancillería. En los proyectos educativos se destacan alianzas con entidades como el Servicio Nacional de Aprendizaje (sENA) y Colciencias, la Universidad de La Salle, la Universidad de Antioquia, la Universidad Nacional de Colombia, la Universidad de San Gil y la Biblioteca Luis Ángel Arango. Mientras que en los programas de microcréditos y desarrollo de procesos productivos existen alianzas con Bancoldex, InNpulsa y Procolombia.

De igual manera, estas fundaciones han trabajado con otras de los sectores de minería e hidrocarburos, con fundaciones que tienen presencia en la misma zona de influencia o con otras que trabajan en áreas temáticas similares. Por ejemplo, las fundaciones que hacen parte de la AFP han colaborado en programas entre ellas, lo cual evidencia una de las ventajas de pertenecer a esta organización; además de tener en cuenta que Ecopetrol es socio fundador de estas, factor que facilita la coordinación. Este tipo de colaboración permite visibilizar la labor de la IsP, y el aprendizaje mutuo hace posible potenciar la contribución de las fundaciones empresariales al cumplimiento de la Agenda 2030.

Si bien las cifras reflejan un comportamiento dinámico de las alianzas de las 
TABLA 2. ALIANZAS DE LAS FUNDACIONES DE MINERÍA E HIDROCARBUROS, POR TIPO DE ACTOR

\begin{tabular}{|c|c|c|c|c|c|c|c|c|}
\hline Fundación & $\begin{array}{l}\text { Sector } \\
\text { Público } \\
\text { (\%) }\end{array}$ & $\begin{array}{c}\text { Academia } \\
(\%)\end{array}$ & $\begin{array}{l}\text { Sector } \\
\text { Privado } \\
(\%)\end{array}$ & $\begin{array}{c}\text { Otras } \\
\text { fundaciones } \\
\text { (\%) }\end{array}$ & $\begin{array}{l}\text { OSC - ONG - } \\
\text { Sociedad } \\
\text { civil (\%) }\end{array}$ & $\begin{array}{c}\text { Organismos } \\
\text { Internacionales } \\
\text { (\%) }\end{array}$ & $\begin{array}{c}\text { Otros } \\
(\%)\end{array}$ & $\begin{array}{c}\text { Total } \\
\text { alianzas }\end{array}$ \\
\hline $\begin{array}{l}\text { Fundación } \\
\text { Oleoductos de } \\
\text { Colombia (FODC) }\end{array}$ & 58,33 & 8,33 & 16,67 & 4,17 & & 12,50 & & 24 \\
\hline $\begin{array}{l}\text { Fundación Alto } \\
\text { Magdalena (FAM) }\end{array}$ & 52,17 & & 26,09 & 4,35 & 8,70 & 8,70 & & 23 \\
\hline $\begin{array}{l}\text { Fundación } \\
\text { Amanecer }\end{array}$ & 37,50 & & 25,00 & 12,50 & 6,25 & 18,75 & & 16 \\
\hline FUNDESMAG & 45 & 20 & 10 & 10 & 10 & & 5 & 20 \\
\hline $\begin{array}{l}\text { Fundación } \\
\text { Alcaraván }\end{array}$ & 77,8 & & 11,1 & & 11,1 & & & 9 \\
\hline $\begin{array}{l}\text { Fundación } \\
\text { Cerrejón }\end{array}$ & 24 & & 65 & & 6 & 6 & & 17 \\
\hline Fundación Terpel & 65,5 & 3,4 & 3,4 & 13,8 & 10,3 & 3,4 & & 29 \\
\hline $\begin{array}{l}\text { Fundación Gases } \\
\text { del Caribe }\end{array}$ & 54 & 15 & 8 & 23 & & & & 13 \\
\hline $\begin{array}{l}\text { Fundación Social } \\
\text { Acerías Paz del } \\
\text { Río }\end{array}$ & 28,57 & 14,29 & 21,43 & 28,57 & 7,14 & & & 14 \\
\hline $\begin{array}{l}\text { Fundación } \\
\text { Surtigas }\end{array}$ & 17,81 & 2,74 & 5,48 & & 64,38 & 8,22 & 1,37 & 73 \\
\hline
\end{tabular}

Nota: los campos en blanco indican que no se encontró información correspondiente a alianzas con este tipo de actor. Fuente: elaboración propia con base en informes de sostenibilidad, páginas web y encuesta realizada.

fundaciones con otros actores, de acuerdo con las directrices de netFWD, también se debería hacer énfasis en sus características y solidez. La construcción de alianzas es un proceso complejo que presenta obstáculos debido a factores como la complejidad en los procesos y tramitología $(80 \%)$, la diferencia de periodos presupuestales o la limitación a presupuestos anuales ( $80 \%$ ), el desconocimiento de la labor social de las fundaciones $(60 \%)$, los espacios de diálogo entre actores se mantienen en niveles consultivos y no son decisorios (60\%), y la poca flexibilidad de los aliados en la gestión de los proyectos $(60 \%)$.
En suma, la colaboración con distintas entidades del orden nacional refleja el papel activo que ejercen las fundaciones en asuntos de desarrollo económico y equidad social en las zonas de influencia, así como su interés por desarrollar programas que mejoren las condiciones de vida de los beneficiarios y contrarresten los efectos negativos (ambientales y socioeconómicos) de la explotación y extracción de minerales e hidrocarburos. Si bien el capital con el que cuentan las fundaciones empresariales es limitado con respecto al del Gobierno nacional, la colaboración con otras organizaciones permite ampliar el alcance y el 
número de beneficiarios y contribuye al cumplimiento de las exigentes metas de la Agenda 2030 (Stott, 2017).

\section{La cooperación internacional, una oportunidad para las fundaciones de los sectores de minería e hidrocarburos}

La cooperación internacional es concebida como una fuente de recursos dentro del esquema de financiamiento de los ods en Colombia (Comisión ods, 2018). La Agencia Presidencial de Cooperación Internacional - APC Colombia, hace parte de la arquitectura institucional impulsada por el Gobierno nacional para la implementación de los oDs en el país, y es la entidad que coordina la cooperación internacional en Colombia y conecta el sector privado con los recursos de cooperación internacional (figura 2).

Para ello, APC Colombia definió tres ejes temáticos en la hoja de ruta 2015-2018: construcción de paz, desarrollo rural sostenible, y conservación y sostenibilidad ambiental, los cuales, a pesar de no ser excluyentes, permitieron focalizar y dinamizar la cooperación que recibe Colombia (APC Colombia, s. f.). Por tanto, uno de los aspectos relevantes al evaluar la posibilidad de acceder a recursos de cooperación internacional es si la temática de los proyectos o programas está alineada con las prioridades de esta Agencia ${ }^{4}$.

\section{FIGURA 2. METODOLOGÍA DE ARTICULACIÓN ENTRE EL SECTOR PRIVADO COLOMBIANO Y LA COOPERACIÓN INTERNACIONAL}

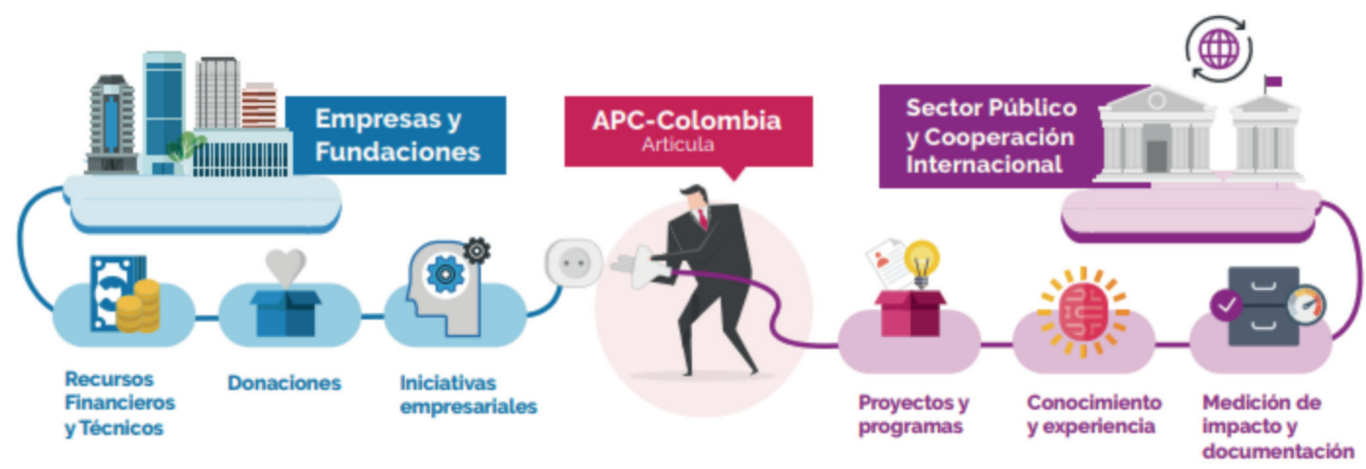

Fuente: Informe de gestión 2017 - APC Colombia, p. 16.

\footnotetext{
4 Al momento de la realización de la presente investigación (junio de 2019) no ha sido publicado el Plan Estratégico Institucional 2019-2022 de APC Colombia, por tanto, no se realizan comentarios sobre posibles convergencias con los ejes estratégicos de las fundaciones.
} 
Este aspecto y otros, como el tipo de cooperación ${ }^{5}$, la modalidad ${ }^{6}$, la presencia geográfica de los cooperantes, entre otros, se deben evaluar al momento de buscar recursos de cooperación internacional. Estos puntos son abordados detalladamente en la guía "Lineamientos de cooperación para el sector privado", de la Fundación Andi y APC Colombia (2016).

Con relación a las fundaciones de los sectores de minería e hidrocarburos, se evidencia que las alianzas con organismos internacionales son las menos recurrentes dentro de su gestión (tabla 2). De acuerdo con los informes de sostenibilidad, las páginas web y la encuesta realizada, el $60 \%$ de las fundaciones que hacen parte de la muestra han establecido al menos un acuerdo de cooperación con organismos internacionales entre el año 2015 y 2018. La Agencia de los Estados Unidos para el Desarrollo Internacional (USAID) es el cooperante más recurrente, principalmente en el marco de los programas de microcrédito y banca comunal. Por su parte, la Fundación de Oleoductos de Colombia y la Fundación Amanecer han recibido cooperación del PNUD en programas de desarrollo productivo y empresarial. La Fundación Cerrejón mantiene una alianza con el Programa Mundial de Alimentos (PMA) para el fortalecimiento de sistemas productivos tradicionales y el desarrollo de un programa de seguridad alimentaria que se lleva a cabo desde 2015. Por su parte, la Fundación Surtigas ha trabajado en colaboración con la Agencia de Cooperación Alemana (GIz) y con la Fundación Suiza de Cooperación al Desarrollo (sWISSAID) en programas de fortalecimiento de capacidades en población juvenil.

En cuanto al tipo de cooperación recibida, no existe información detallada en los informes de gestión o sostenibilidad de las fundaciones, sin embargo, se observa que la cooperación financiera (reembolsable y no reembolsable) y la cooperación técnica son las tipologías más utilizadas en las relaciones con las fundaciones de los sectores de minería e hidrocarburos.

A pesar de que las alianzas mencionadas no son las únicas existentes entre fundaciones de los sectores de minería e hidrocarburos y organismos internacionales, reflejan que estas relaciones son menos recurrentes con respecto a alianzas con entidades de orden nacional. Entre los factores que limitan la cooperación con entidades internacionales se destacan las contrapartidas en recursos -en proporción $1: 1$, requeridas por los cooperantes-, ya que en algunos casos las fundaciones empresariales no cuentan con estos recursos y las empresas que las financian tampoco cuentan con un presupuesto destinado para este fin. Por ejemplo, en 2016, la Fundación Alcaraván solicitó recursos de la Embajada Alemana en el marco de un proyecto de fortalecimiento de cultivo de cacao, sin embargo, el proyecto no fue aprobado, ya que la fundación no contaba

\footnotetext{
5 Tipos de cooperación de acuerdo con APC Colombia: técnica, financiera reembolsable, financiera no reembolsable, ayuda humanitaria, asistencia alimentaria y cooperación cultural.

6 Modalidades de cooperación según tipología de APC Colombia: Ayuda oficial al desarrollo, Cooperación SurSur, Cooperación Col-Col, Cooperación triangular y Donaciones en especie.
} 
con las contrapartidas exigidas (Fundación El Alcaraván, 2016).

Otro factor que ha limitado el acceso a recursos de cooperación es la complejidad en los procesos burocráticos. El sistema nacional de cooperación internacional está diseñado a partir de un enfoque territorial, en el que las iniciativas de cooperación de las fundaciones deben realizarse vía comités de cooperación regional o departamental; sin embargo, existen debilidades en la institucionalidad territorial que impiden llegar a acuerdos para acceder a recursos de cooperación internacional, inquietud manifestada por las fundaciones Alcaraván y Cerrejón en 2015 (AfeCol, 2015).

La información encontrada con respecto a cooperación internacional solo hace referencia a recursos (financieros y técnicos) recibidos por parte de las fundaciones de los sectores de minería e hidrocarburos; en las fuentes consultadas no hay mención de cooperación otorgada por estas fundaciones hacia otras organizaciones de nivel internacional.

En definitiva, a pesar de que las alianzas con organismos internacionales son las menos recurrentes, acceder a recursos de cooperación internacional representa una oportunidad para las fundaciones en la medida en que les permite ampliar la cobertura de sus programas y proyectos, beneficiarse del conocimiento y experiencia de otros actores de desarrollo, les otorga reconocimiento a nivel internacional y les brinda la oportunidad de compartir experiencias exitosas que pueden llegar a replicarse en otros territorios.

\section{CONCLUSIONES}

En la estrategia de implementación de los ods en Colombia, se planteó la necesidad de integrar nuevos actores como el sector privado y la sociedad civil, con el objetivo de tener un mayor alcance en los programas de desarrollo social, económico y ambiental. Asimismo, el esquema de financiación de esta agenda tiene en cuenta recursos provenientes tanto del sector privado como de cooperación internacional, dada la magnitud de las metas y los recursos limitados del Estado colombiano.

A diferencia de otros análisis realizados sobre el aporte del sector privado y social al cumplimiento de los oDs, este enfatiza en el trabajo realizado por las fundaciones empresariales de los sectores de minería e hidrocarburos, su compromiso con el bienestar social de las poblaciones que hacen parte de sus zonas de influencia y los factores que limitan su alcance.

Este estudio encuentra que las fundaciones del sector de minería e hidrocarburos responden a las temáticas abordadas en el ámbito social de los oDs. Así, nueve de diez fundaciones han realizado programas que tienen por objetivo disminuir la pobreza y las desigualdades (Objetivo 1); nueve de diez fundaciones llevaron a cabo programas educativos (Objetivo 4); seis de diez implementaron programas de igualdad de género (Objetivo 5) ya sea de manera transversal o a partir de programas específicos. Mientras que los programas relacionados con erradicación del hambre (Objetivo 2) y con salud y bienestar (Objetivo 3) fueron los menos recurrentes. 
A pesar de que los programas no se formularon en función de los oDs, hoy en día existe un conocimiento, interés y apropiación de la Agenda 2030 por parte de las fundaciones y, por tanto, una oportunidad de articular su gestión con estos objetivos. No obstante, su aporte no se ha medido en relación directa con las metas e indicadores de los oDs y no se han implementado métodos de medición y reporte homogéneos, aspectos que son necesarios para visualizar la labor de las fundaciones y para alinear sus esfuerzos con la hoja de ruta implementada por el Gobierno nacional.

El aporte de las fundaciones también se mide en términos de fortalecimiento de alianzas y cooperación con otros actores, por lo cual se destaca que la totalidad de las fundaciones que hacen parte de la muestra han llevado a cabo alianzas con gobiernos locales, regionales y entidades del sector público en general. Las alianzas con el sector privado ocupan el segundo lugar en frecuencia, seguidas de alianzas con la academia y con otras fundaciones. Sin embargo, las alianzas con organismos internacionales son las menos recurrentes, debido a la complejidad y el desconocimiento de los procesos, a la falta de articulación entre actores y ejes estratégicos y a las prioridades de las fundaciones.

A pesar de ello, las alianzas con organismos internacionales y el acceso a recursos de cooperación internacional representan una oportunidad para que las fundaciones amplíen la cobertura de sus programas, se beneficien del conocimiento y la experiencia de otros actores de desarrollo, y visualicen y compartan experiencias exitosas que puedan ser replicadas en otros escenarios.
En definitiva, los primeros años de la implementación de la Agenda 2030 para el Desarrollo Sostenible, una agenda de desarrollo internacional, son claves para articular los roles de los actores y a fin de establecer los recursos necesarios para su cumplimiento. Dado el liderazgo del Gobierno nacional y la institucionalidad que ha puesto a disposición para su implementación, las fundaciones empresariales del sector de minería e hidrocarburos tienen oportunidades para ampliar su aporte al cumplimiento de los ons y trabajar en colaboración con organismos del orden local, nacional o internacional.

No obstante, por la naturaleza misma de las fundaciones empresariales, el alcance de sus acciones desarrolladas a través de la inversión social privada es limitado en la medida en que corresponden a acciones concretas en las zonas de influencia. En este sentido, el aporte de las fundaciones, y del sector privado en general, juega un papel complementario en el desarrollo sostenible.

\section{REFERENCIAS}

AfeCol (2015). APC-Colombia busca crear alianzas con el sector privado-AFE. Recuperado de https://www. youtube.com/watch?v=aAwzyx $8 \mathrm{~h} 5$ So

Agencia de Cooperación Alemana (GIz) (s. f.). Colombia. Recuperado de https://www.giz.de/en/worldwide/29848.html

APC Colombia y Fundación ANDI (2016). Lineamientos de cooperación internacional para el sector privado. Recuperado de http://www.apccolombia.gov. co/sites/default/files/archivos_usuario/publicaciones/guia-cooperacion-20sep-flipboard.pdf 
Arteaga, D. (2015). El desarrollo humano y la generación de capacidades. Diálogos, 5, 47-57.

Asociación de Fundaciones Familiares y Empresariales (AFE) (2018). Información corporativa. Recuperado de https://afecolombia.org/la-afe/

Ayllón, B. (2007). La Cooperación Internacional para el Desarrollo: fundamentos y justificaciones en la perspectiva de la teoría de las relaciones internacionales. Carta Internacional, 2(2), 25-40.

Cassand, M. (2014). Análisis de la RSE de dos grandes compañias petroleras: contraste entre las acciones realizadas y la percepción pública. Madrid: Universidad Pontificia Comillas. Recuperado de https://repositorio.comillas.edu/xmlui/ bitstream/handle/11531/449/TFG000423. pdf?sequence $=1$

Comisión ods (2018). Reporte nacional voluntario 2018. Departamento Nacional de Planeación. Bogotá: Comisión ods.

Comisión ods (2019). Explorador de datos, tasa de mortalidad materna. Recuperado de https:// www.ods.gov.co/es/data-explorer?state $=\% 7 \mathrm{~B} \%$ 22 goal $\% 22 \% 3 \mathrm{~A} \% 223 \% 22 \% 2 \mathrm{C} \% 22$ indicator \%22\%3A\%223.1.1.G\%22\%2C\%22dimensio n\%22\%3A\%22DES_GEO_DEPTOS\%22\%2C\%2 2view\%22\%3A\%22bar\%22\%7D

Departamento Administrativo Nacional de Estadísticas (DANE) (2019). Pobreza Monetaria y Multidimensional en Colombia - Año 2018. Boletín técnico. Recuperado de https://www.dane.gov. co/files/investigaciones/condiciones_vida/pobreza/2018/bt_pobreza_multidimensional_18.pdf

Departamento Nacional de Planeación (DNP) (2018a). Documento CONPES 3918. Estrategia para la implementación de los Objetivos de Desarrollo Sostenible en Colombia (ODS). Bogotá: DNP.

Departamento Nacional de Planeación (DNP) (2018b). The private sector and its contribution to the SDGs:
Ajourney to data gathering through corporate sustainability reporting in Colombia. Recuperado de http://www.odsreportevoluntario.org/ Private\%20Sector\%20and\%20Its\%20Contribution $\% 20$ to $\% 20$ the $\% 20$ sDGs-\%20A\%20 Journey\%20to\%20Data\%20Gathering $\% 20$ and $\% 20$ Reporting\%20in\%20Colombia $\% 20$ DigitaL.pdfFundación Cinep (2014). Las tendencias de la sociedad civil en Colombia. Recuperado de https://issuu.com/afpetroleo/docs/ cartilla_afp_tendencias_soc._civil_

Fundación DIs y Fundación Promigas (2012). Las fundaciones empresariales en Colombia: una mirada a su estructura y dinámicas. Recuperado de https://grupodis.net/docs_gestrategica/ Presentaci\%C3\%B2n\%20Fundaciones\%20 Empresariales\%20en\%20Colombia_Fund_ DIs_2012.pdf

Fundación El Alcaraván (2016). Informe de Gestión primer semestre de 2016. Recuperado de https://issuu.com/elalcaravan/docs/informe_de_gesti_n_2016-1

Gallopín, G. (2003). Sostenibilidady desarrollo sostenible: un enfoque sistémico. Santiago de Chile: CEPAL.

García, J. y Salcedo, N. (2017). Inversiones de impacto como puente entre la cooperación internacional y el sector privado: posconflicto en Colombia. Revista Española de Desarrollo y Cooperación, (40), 105-116.

Hernández Sampieri, R. (2010). Metodología de la investigación (5 ed.). México: McGraw-Hill.

Malagón, J. (2016). La competitividad del sector de hidrocarburos en las diferentes regiones de Colombia. Bogotá: Cuadernos PNUD.

Ministerio de Salud y Protección Social (2018). Análisis de Situación de Salud (ASIS), Colombia 2017. Recuperado de https://www.minsalud.gov.co/ sites/rid/Lists/BibliotecaDigital/RIDE/Vs/ED/ PSP/asis-nacional-2017.pdf 
Montoya, A. (2018). APC y AFE buscan mejorar articulación entre cooperación internacional e inversión social privada. Asociación de Fundaciones Empresariales. Recuperado de https://afecolombia. org/apc-y-la-afe-buscan-articular-cooperacioninternacional-con-inversion-social-privada/

Mora, M. y Martínez, F. (2018). Desarrollo local sostenible, responsabilidad social corporativa y emprendimiento social. Equidad y DesarroLlo, (31), 27-46. Recuperado de http://dx.doi. org/10.19052/ed.4375

Observatorio de Asuntos de Género (s. f.). Tasa de mortalidad materna por departamento. Recuperado de http://obs.equidadmujer.gov.co/Observatorio/ Observatorio.aspx?rpt=ZqZi5sfrhbapxtkwe/ $\mathrm{M} 6 \mathrm{pQ}==$

OCDE netFWD (2016). Colaboración entre fundaciones y el gobierno: evidencias desde México. Paris: Centro de Desarrollo de la OCDE.

ONU-Mujeres (2018). El progreso de las mujeres en Colombia 2018: transformar la economía para realizar los derechos. Colombia: ONU-Mujeres.

Pauselli, G. (2013). Teorías de relaciones internacionales y la explicación de la Ayuda Externa. Revista Iberoamericana de Estudios de Desarrollo, (2), 72-92.

Pintor-Pirzkall, H. (2017). Las alianzas público-privadas en la cooperación alemana actual: el programa developpr. Revista Española de Desarrollo y Cooperación, (40), 67-75.

Programa de las Naciones Unidas para el Desarrollo (PNUD) (s. f.). Agenda 2030 para el desarrollo sostenible. Recuperado de https://www.undp. org/content/undp/es/home/2030-agenda-forsustainable-development.html
Rudas, G. y Espitia, J. (2013). La paradoja de la minería y el desarrollo. Análisis departamental y municipal para el caso de Colombia. Minería en Colombia. Institucionalidad y territorio, paradojas y conflictos. Bogotá: Contraloría General de la República. Imprenta Nacional de Colombia.

Ruiz, P. (2017). Jeffrey Sachs (2014). La era del desarrollo sostenible (reseña). Oasis, (26), 159-164. Recuperado de https://doi.org/10.18601/16577558. n26.11

Sachs, J. (2014). The Age of Sustainable Development. New York: Columbia University Press.

Salas, R. (2017). Un análisis crítico al marco conceptual de desarrollo sostenible y sus herramientas de medición. Revista En-Contexto, 6(8), 171-184. Recuperado de http://ojs.tdea.edu.co/index. php/encontexto/article/view/485/648

Stott, L. (2017). Partnership fundamentals: Understanding organisational incentives. Revista Española de Desarrollo y Cooperación, (40), 27-37.

Vargas, J. G. (2008). Análisis crítico de las teorías del desarrollo económico. Economía Gestión y Desarrollo, (6), 109-131.

Vergara, C. y Ortiz, D. (2016). Desarrollo sostenible: enfoque desde las ciencias económicas. Apuntes del CENES, 35(62), 15-52.

Villar, R. (2015). Recursos privados para la transformación social. Filantropia e inversión social privada en América Latina hoy. Bogotá D.C.: AFE, BID, Avina, Cemefi, GDFE y GIFE.

Villar, R. (2018). Las fundaciones en Colombia. Características, tendencias, desafíos. Bogotá: AFE. 